\title{
André Lichnerowicz Prize in Poisson Geometry 2018
}

Eva Miranda (Universitat Politecnica de Catalunya, Barcelona, Spain)

The André Lichnerowicz Prize in Poisson geometry was established in 2008. It is awarded for notable contributions to Poisson geometry, every two years at the International Conference on Poisson Geometry in Mathematics and Physics, to researchers completing their doctorates at most eight years before the year of the conference.

The prize is named in memory of André Lichnerowicz (1915-1998), whose work was fundamental in establishing Poisson geometry as a branch of mathematics. It is awarded by a jury composed of members of the scientific/advisory committee of the conference.

The prize for the year 2018 was awarded to:

\section{Brent Pym and Chelsea Walton}

on 16 July 2018 at the Fields Institute, Toronto

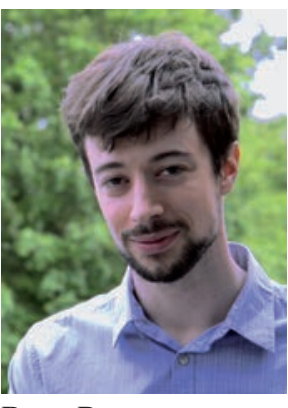

Brent Pym received his $\mathrm{PhD}$ at the University of Toronto in 2013, under the direction of Marco Gualtieri. He has held postdoctoral positions at McGill, Oxford and Edinburgh and recently accepted an assistant professorship in the Department of Mathematics and Statistics at McGill University. In his thesis work, Pym classified the noncommutative deformations of complex projective 3-space, proved the 4-dimensional case of the Bondal conjecture about Fano Poisson manifolds and, jointly with Gualtieri and Li, developed the theory of Stokes groupoids on Riemann surfaces. In recent work, Pym developed the notion of an elliptic singularity for a holomorphic Poisson structure and used it to obtain some of the only available classification results in dimension greater than three. He has also developed the notion of a holonomic Poisson manifold (jointly with Schedler), bringing the theory of perverse sheaves into the mainstream of Poisson geometry. In additional joint works, Pym has contributed to the enumerative geometry of noncommutative spaces and to the theory of Dirac structures and Courant algebroids as objects in shifted symplectic geometry.

Chelsea Walton Chelsea Walton completed her $\mathrm{PhD}$ in 2011 at the University of Michigan, under the direction of Toby Stafford and Karen Smith. Following postdoctoral stays at the University of Washington, MSRI and MIT, she took on an assistant professorship at Temple Univer-

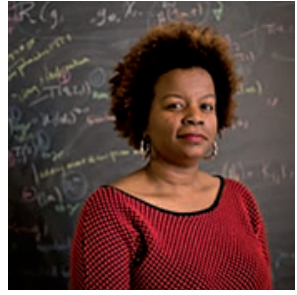

Chelsea Walton (picture by Ryan Brandenburg) sity in Philadelphia in 2015. In July 2018, she joined the Mathematics Department at the University of Illinois at Urbana-Champaign as an associate professor with tenure. Walton has written several important works in Poisson geometry, in addition to being a well-established expert in noncommutative algebra and quantum groups. Her work in Poisson geometry includes a deep investigation of the 3-dimensional and 4-dimensional Sklyanin algebras, especially those that are module-finite over their centre. Jointly with Wang and Yakimov, Walton showed that these are close analogues of Poisson algebras, namely Poisson Z-orders, which carry Poisson structures on the centre. Walton, in joint work with several collaborators, has written a deep series of works on actions of Hopf algebras on commutative and noncommutative domains, showing that semisimple Hopf actions generally factor through group algebra actions, also investigating the difficult non-semisimple case. She also gave a negative answer to the longstanding conjecture about whether the universal enveloping algebra of the Witt algebra is noetherian (jointly with Sierra).

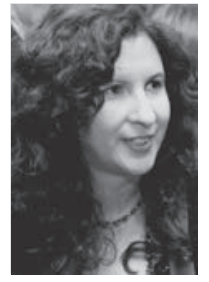

Eva Miranda [eva.miranda@upc.edu] is an ICREA Academia Professor at Universitat Politècnica de Catalunya, an Honorary Doctor Vinculado at ICMAT-Madrid and a Chercheur Affilié at Observatoire de Paris. Eva Miranda is the Director of the Laboratory of Geometry and Dynamical Systems at UPC and a member of the Governing Board of the Barcelona Graduate School of Mathematics. In 2017, she was awarded a Chaire d'Excellence of the Fondation Sciences Mathématiques de Paris and an ICREA Academia Prize. Eva Miranda's research is at the crossroads of differential geometry, mathematical physics and dynamical systems. Her area of expertise is symplectic and Poisson geometry. She works with objects at the interface of geometry and physics such as integrable systems and group actions acquainting for symmetries of the systems. Her research deals with geometric and dynamic aspects of the singularities arising in symplectic and Poisson manifolds, as well as with mathematical models for their quantisation. During the period 2010-2018, she has been a member of the Editorial Board of the Newsletter of the EMS. 